\title{
Spectral Structure of Critical Opalescence: Binary Mixture
}

\author{
Raymond D. Mountain*
}

(June 29, 1965)

\begin{abstract}
The linearized hydrodynamic equations of irreversible thermodynamics are used to obtain the time dependence of the $k$ th Fourier component of a concentration fluctuation in the one-phase region of a binary mixture. The frequency of the light scattered by concentration fluctuations near the critical mixing point is found to be broadened into a Lorentzian distribution with a half-width proportional to the mass diffusion coefficient, $D$. The possibility of detecting space dispersion in $D$ is discussed.

Key Words: Binary diffusion coefficient, concentration fluctuations, critical opalescence, gas laser, optical hetrodyne system, spectral distribution of scattered light.
\end{abstract}

\section{Introduction}

According to the Einstein [1] ${ }^{1}$-Smoluchowski [2] theory of light scattering in a fluid, scattering results from fluctuations in the dielectric constant in small volumes of the fluid. Since static inhomogeneities do not exist in fluids, these fluctuations vary in time and this results in a spectrum of frequencies of the scattered light [3].

Critical opalescence refers to the anomalously large amount of scattering which occurs in the vicinity of a critical point [4]. In this paper we are concerned with the spectrum of critical opalescence in the one phase region of a binary mixture. Here the fluctuating quantity is the concentration of one of the components of the mixture [5]. Large fluctuations in the concentration are possible near the critical mixing point because the chemical potentials of the components vary quite slowly with concentration in that region [6].

Although the spectrum of the scattered light is confined to a narrow range of frequencies, it is now possible to investigate the spectrum using a gas laser and an optical heterodyne system [7]. This type of system has been used to examine critical opalescence in a cyclohexane and aniline mixture [8].

\section{Formulation of the Problem}

We shall determine the time dependence of concentration fluctuations by calculating the decay of a fluctuation in time using the linearized equations of irreversible thermodynamics [9]. For a binary mixture, these are the continuity equation

$$
\partial \rho / \partial t+\rho_{0} \operatorname{div} \mathbf{v}=0,
$$

*NAS-NRC postdoctoral Fellow at the National Bureau of Standards.

${ }^{1}$ Figures in brackets indicate the literature references at the end of this paper. the Navier-Stokes equation (only the longitudinal part of $\mathbf{v}$ is included)

$$
\rho_{0} \partial \mathbf{v} / \partial t=-\operatorname{grad} p+\left(\frac{4}{3} \eta_{s}+\eta_{B}\right) \operatorname{grad} \operatorname{div} \mathbf{v},
$$

the energy transport equation

$$
\begin{aligned}
\rho_{0} c_{p} \partial T / \partial t-\rho_{0} k_{T}(\partial \mu / \partial c)_{P, T} \partial c / \partial t \\
\\
\quad+\rho_{0} T_{0}(\partial S / \partial p)_{T, c} \partial p / \partial t=\kappa \nabla^{2} T,
\end{aligned}
$$

and the equation of continuity for the concentration

$\rho_{0} \partial c / \partial t=\rho_{0} D\left[\nabla^{2} c+\left(k_{T} / T_{0}\right) \nabla^{2} T+\left(k_{p} / p_{0}\right) \nabla^{2} p\right]$.

In these equations, $\rho$ is the density, $T$ is the temperature, $p$ is the pressure and $c$ is the concentration; equilibrium values are denoted by a subscript zero $\left(\rho_{0}\right)$. Other quantities are the thermal conductivity $\kappa$, the shear and bulk viscosities $\eta_{s}$ and $\eta_{B}$, the diffusion coefficient $D$, the thermal diffusion ratio $k_{T}$ and the barodiffusion ratio $k_{p}$. The thermodynamic derivatives involve the entropy $S$ and the chemical potential $\mu$ of the mixture. The notation is that of reference 9 .

A light scattering experiment provides a Fourier analysis of the fluctuations in the scattering medium. If both the scattering angle and the frequency of the scattered light are measured precisely, both the space and the time variation are Fourier analyzed [3]. Using the hydrodynamic equations, eqs ( 1 to 4 ), we can determine the time dependence of the $k$ th Fourier component, $c(k, t)$, of a concentration fluctuation in terms of an initial fluctuation $c(k)$. The intensity of the scattered light is proportional to $\langle c(k, \omega) c(-k)\rangle$ where the $\langle\ldots\rangle$ indicate an equilibrium average over initial $(t=0)$ values and

$$
c(k, \omega)=2 \operatorname{Re} \int_{0}^{\infty} c(k, t) e^{i \omega t} d t .
$$


In eq (5) $k=2 k_{i} \sin \theta / 2$ is the change in the wave vector which occurs when light with wave vector $k_{i}$ in the medium is scattered through an angle $\theta$. The change in the angular frequency is denoted by $\omega$.

It is a straightforward, if involved, procedure using eqs ( 1 to 4 ) to obtain $c(k, t)$ in terms of the initial fluctuation $c(k)$ [10]. As expected, two propagating (phonon) modes and two diffusive modes are obtained. The diffusive modes are a combination of thermal diffusion and mass diffusion. Near the critical mixing point, where $c_{p}$ is anomalously large and $D$ is small [11], the situation is appreciably simpler. There eqs (3) and (4) may be used to describe the diffusive modes. The pressure terms may be neglected because the pressure is insensitive to the local concentration in the vicinity of the critical point [6].

In the next section, we shall obtain an expression for the spectral structure of critical opalescence using the constant pressure versions of eqs (3) and (4). The results are identical to those obtained using the full set of equations.

\section{Calculation}

The equations governing the temperature and the concentration are

$$
\partial T / \partial t-k_{T} / c_{p}(\partial \mu / \partial c)_{P, T} \partial c / \partial t=\left(\kappa / \rho_{0} c_{p}\right) \nabla^{2} T
$$

and

$$
\partial c / \partial t=D\left[\nabla^{2} c+\left(k_{T} / T_{0}\right) \nabla^{2} T\right] .
$$

The first step is to obtain the Fourier (space) and Laplace (time) transforms of eqs (6) and (7). The transform of the concentration is

$$
c(k, s)=\int_{v} \int_{0}^{\infty} e^{-i \mathbf{k} \cdot \mathbf{r}} e^{-s t} c(r, t) d \mathbf{r} d t .
$$

Equations (6) and (7) become

$$
\begin{array}{r}
s T(k, s)-s k_{T} / c_{p}(\partial \mu / \partial c)_{P, T} c(k, s)+\left(\kappa k^{2} / \rho_{0} c_{p}\right) T(k, s) \\
=T(k)-k_{T} / c_{p}(\partial \mu / \partial c)_{P, T} c(k)
\end{array}
$$

and

$$
s c(k, s)+D k^{2} c(k, s)+\left(D k_{T} k^{2} / T_{0}\right) T(k, s)=c(k) .
$$

Neglecting $T(k)$ because $\langle\Delta T \Delta c\rangle=0$ [12], we obtain

$$
\frac{c(k, s)}{c(k)}=\frac{s+\chi k^{2}+\xi k^{2}}{s^{2}+s k^{2}(\chi+D+\xi)+\chi D k^{4}}
$$

where

$$
\chi=\kappa / \rho_{0} c_{p}
$$

and

$$
\xi=\left(D k^{2} / c_{p} T_{0}\right)(\partial \mu / \partial c)_{P, T} .
$$

From this it follows that

$$
\begin{gathered}
\frac{c(k, t)}{c(k)}=\frac{\frac{1}{2} \mathscr{D}+\frac{1}{2}(\chi-D)+\frac{1}{2} \xi}{\mathscr{D}} \exp \left[-\frac{1}{2}(\mathscr{C}-\mathscr{D}) k^{2} t\right] \\
+\frac{-\frac{1}{2} \mathscr{D}+\frac{1}{2}(\chi-D)+\frac{1}{2} \xi}{\mathscr{D}} \exp \left[-\frac{1}{2}(\mathscr{C}+\mathscr{D}) k^{2} t\right]
\end{gathered}
$$

where

$$
\mathscr{D}=\left[(\chi-D)^{2}+2(\chi+D) \xi+\xi^{2}\right]^{1 / 2}
$$

and

$$
\mathscr{C}=\chi+D+\xi
$$

Near the critical point both $\chi$ and $D(\chi>D)$ are small [11] so that

$$
\mathscr{D} \approx \chi-D
$$

and eq (12) simplifies to

$$
\frac{c(k, t)}{c(h)}=\mathrm{e}^{-D k^{2 t}}+\varphi\left(D^{2}\right) .
$$

Equation (14) is based on the reasonable assumption that $(\chi-D)^{2}$ vanishes more slowly than $D^{2} / c_{p}$. Combining eqs (5) and (14) we find that

$$
\langle c(k, \omega) c(-k)\rangle=\langle c(k) c(-k)\rangle \frac{2 D k^{2}}{\left(D k^{2}\right)^{2}+\omega^{2}} .
$$

An equation equivalent to eq (14) has been derived by Debye [14]. His derivation is based on the assumption that there is no dynamical coupling between the concentration and the temperature. We have shown this to be the case when $D<\chi$ and both quantities are small. Then it is possible to separate the two diffusive modes into one dominated by the thermal diffusity $\chi$ and one dominated by the diffusion coefficient $D$. The mode dominated by the thermal diffusity has effectively zero weight in the sum rule, eq (16), for the intensity of the scattered light.

$$
\frac{1}{2} \frac{1}{\pi} \int_{-\infty}^{\infty}\langle c(k, \omega) c(-k)\rangle d \omega=\left\langle c(k) c\left(-k_{i}\right)\right\rangle .
$$

(The phonon modes, which we have not explicitly considered, also have zero weight.)

If only the intensity of the scattered light is observed, $\langle c(k) c(-k)\rangle$ is obtained. Near the critical point $\langle c(k) c(-k)\rangle$ is commonly assumed to be of the form $\left(k^{2}+\kappa^{2}\right)^{-1}$ where $\kappa^{-1}$ is the range of the two particle correlation function [13]. Away from the critical point, $\langle c(k) c(-k)\rangle$ is independent of the wave vector and equals the mean square fluctuation in the concentration. 


\section{Discussion}

The spectrum of critical opalescence is that of a Lorentzian distribution with a half width of $D k^{2} / \pi$ cycles/sec. Recent measurements of the spectrum of critical opalescence in a mixture of cyclohexane and aniline are of this type [8]. Measurements of the spectral structure of critical opalescence provide a gradient-free method of determining the diffusion coefficient.

The type of calculation presented above is certainly appropriate when the range of the two particle correlations is small compared to the wavelength of the light which is scattered. Near the critical point, the range of the correlations is on the order of the wavelength of visible light. It is not unreasonable to expect that near the critical point, transport coefficients may reflect the long range of these correlations by becoming $k$-dependent [15]. This interesting possibility could be checked by seeing whether or not the half width of the Lorentzian distribution varies linearly with $k^{2}$. Any deviation from linearity would suggest that the diffusion coefficient $D$ should be replaced by $D(k)$, a $k$-dependent diffusion coefficient.

(Paper 69A6-373)

\section{References and Notes}

[1] A. Einstein, Ann. Physik 33, 1275 (1910).

[2] M. V. Smoluchowski, Ann. Physik 25, 205 (1908).

[3] L. D. Landau and E. M. Lifshitz, Electrodynamics of Continuous Media ch. 14 (Reading, Mass.: Addison-Wesley Publishing Co., 1960).

[4] P. Debye, J. Chem. Phys. 31, 680 (1959); B. Chu, J. Chem. Phys. 41, 226 (1964) and the references contained in this paper.

[5] H. C. Brinkman and J. J. Hermans, J. Chem. Phys. 17, 574 (1949); John G. Kirkwood and Richard J. Goldberg, J. Chem. Phys. 18, 54 (1950); W. H. Stockmayer, J. Chem. Phys. 18, $58,(1950)$.

[6] J. R. Partington, An Advanced Treatise on Physical Chemistry, Vol. IV, pp. 588-591 (New York: Longmans, Green and Co., Inc., 1953).

[7] H. Z. Cummins, N. Knable, and Y. Yeh, Phys. Rev. Letters 12. 150 (1964).

[8] Y. Yeh, S. S. Alpert, E. Lipworth, L. Seigel, and D. Balzarini, Bull. Am. Phys. Soc. 10,310 (1965); S. Alpert, Y. Yeh, and E. Lipworth, Phys. Rev. Letters 14, 486 (1965).

[9] L. D. Landau and E. M. Lifshitz, Fluid Mechanics, pp. 219-226 (Reading, Mass., Addison-Wesley Publishing Co., 1959).

[10] This program has been carried out by the author.

[11] I. R. Krichevskii, N. E. Khazanova, and Yu. V. Tshkhanskaza, Russ. J. P. Chem. 34, 598 (1960); G. Jura, D. Fraga, G. Maki. and J. H. Hildebrand, Proc. Natl. Acad. Sci. U.S. 39, 19 (1953).

[12] L. D. Landau and E. M. Lifshitz, Statistical Physics, p. 362 (Reading, Mass.: Addison-Wesley Publishing Co., 1958).

[13] There is some experimental evidence that this Ornstein-ZernikeDebye result for the correlation function is not applicable very near the critical point. D. McIntyre, A. Wims, and M. S. Green, J. Chem. Phys. 37, 3019 (1962); B. Chu and W. P. Kao, J. Chem. Phys. 42, 2608 (1965).

[14] P. Debye, Phys. Rev. Letters 14, 783 (1965).

[15] Some of the effects of $k$-dependence have been discussed by Marshall Fixman, J. Chem. Phys. 36, 310 (1962). 